Präv Gesundheitsf 2012 · 7:85-86

DOI 10.1007/s11553-012-0337-2

Online publiziert: 4. April 2012

(c) Springer-Verlag 2012

\title{
V. Kurtz
}

Kriminologisches Forschungsinstitut Niedersachsen e.V., Hannover

\section{Prävention und} Gesundheitsförderung für alle?

\section{Wie spezifische Zielgruppen erreicht werden können und welche Maßnahmen wirken}

Präventives und gesundheitsförderliches Handeln kann nicht nur kurz- und langfristige positive Wirkungen erzielen, sondern darüber hinaus auch (Mehr-)Kosten im Gesundheitswesen einsparen, die bei fehlender oder zu spät einsetzender Prävention und Gesundheitsförderung notwendig werden. Viele Studien belegen die Wirksamkeit diverser früh ansetzender Präventionsmaßnahmen sowohl für Kinder als auch für Erwachsene. Studienergebnisse zur Prävention und Gesundheitsförderung zeigen aber auch, dass nicht alle Adressaten gleich ansprechbar für präventive Maßnahmen sind und denselben Nutzen aus Angeboten ziehen. Die Beiträge der Autorinnen und Autoren in diesem Heft beleuchten sowohl unterschiedliche Wege der Erreichbarkeit wie auch die differentielle Umsetzung und Wirksamkeit von Maßnahmen in den verschiedensten Altersgruppen und Settings von Prävention und Gesundheitsförderung.

Beginnend im frühest möglichen Altersbereich präsentieren Makowsky und Schücking Präventions- und Gesundheitsförderungsmöglichkeiten für adipöse Schwangere, die wiederum auch dem ungeborenen Kind zugute kommen. Allerdings zeigen die Ergebnisse Optimierungsbedarf für eine gezielte Betreuung und Beratung adipöser Schwangerer durch Hebammen und Ärzte auf. Kurtz et al. stellen ebenfalls Forschungsergebnisse aus einem früh ansetzenden Präventionsbereich für die spezielle Zielgruppe von schwangeren Frauen bzw. Müttern mit Migrationshintergrund vor. Sie verdeutlichen, wie die Zielgruppe im Rahmen der Frühen Hilfen erreicht werden kann und welche Maßnahmen für eine erfolgreiche Umsetzung erforderlich sind. Auch Santos-Hövener und Unger greifen die Zielgruppe von Personen mit Migrationshintergrund auf. In ihrem Beitrag untersuchen sie die Möglichkeiten kultursensibler HIV/STI-Prävention bei Sexarbeiterinnen mit Migrationshintergrund in Deutschland und betonen die Wichtigkeit von Peers der jeweiligen Community sowie niedrigschwellig angesiedelte Maßnahmen für eine wirkungsvolle Prävention. Die generelle Wirksamkeit präventiver Evaluationen wird von Buth und Kalke thematisiert. Ihre Literaturübersicht internationaler Studien belegt, dass sowohl universelle als auch selektive Präventionsmaßnahmen im Bereich des Glücksspiels empirisch wirksam sein können. Jordan widmet sich in ihrem Beitrag der Frage, welchen Beitrag das Gesundheits-, Interventions-, Medien- oder Politikmonitoring zur Weiterentwicklung von Prävention und Gesundheitsförderung leisten können.

Dem Alter der Zielgruppen präventiver Maßnahmen folgend stellt nach dem 
frühkindlichen Bereich die Schule einen wichtigen Ort von Präventions- und Gesundheitsförderungsaktivitäten dar. Shamsul und Borrmann stellen Ergebnisse zur Wirksamkeit eines gesundheitsförderlichen Projekts an Grundschulen vor und können v. a. positive Effekte für die Bewegung und gesündere Ernährung der Kinder nachweisen. Wahl et al. präsentieren das universale Präventionsprogramm LARS\&LISA, das von Lehrern durchgeführt wird und bei der Zielgruppe sozial benachteiligter Jugendlicher positive Effekte erzielen kann.

Mit fortschreitendem Alter nehmen die Arbeitswelt und die damit verbundenen Belastungen an Bedeutung auch für die Prävention und Gesundheitsförderung zu. Beck et al. machen darauf aufmerksam, dass in Gefährdungsbeurteilungen zum Arbeitsschutz den psychischen Belastungen immer noch ein zu geringer Stellewert beigemessen wird. Hey et al. stellen die Bedeutsamkeit von aktiven, bewegungsorientierten Regenerationspausen während der Arbeit für die menschliche Arbeitsgedächtnisleistung und Befindlichkeit heraus. NagelPrinz und Paulus wiederum verdeutlichen mithilfe der Ergebnisse einer qualitativen Interviewstudie mit Kita-Leitungen, dass sich neben vielfachen Stressoren auch Ressourcen und Möglichkeiten der Gesundheitsförderung im Setting Kita ergeben.

In einem weiteren Beitrag widmen sich Ose et al. der Bedeutung und Verfügbarkeit schriftlicher Patienteninformationen aus Sicht von Krankenhausmitarbeitern, die sowohl für die Betroffenen als auch für den Klinikalltag an Bedeutung gewinnen.

Insgesamt erhellen die in diesem Heft vorliegenden Beiträge die Bedeut- und Wirksamkeit von Präventions- und Gesundheitsförderungsmaßnahmen in verschiedenen Alters- und Lebensbereichen und tragen somit zur wissenschaftlichen und praktischen Wissenserweiterung bei. Die genaue Betrachtung der Erreichbarkeit unterschiedlicher Zielgruppen und des differentiellen Nutzens von Maßnahmen der Prävention und Gesundheitsförderung stellt auch zukünftig eine lohnende Aufgabe dar.
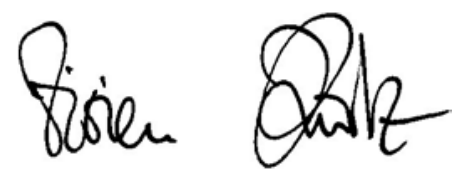

V. Kurtz

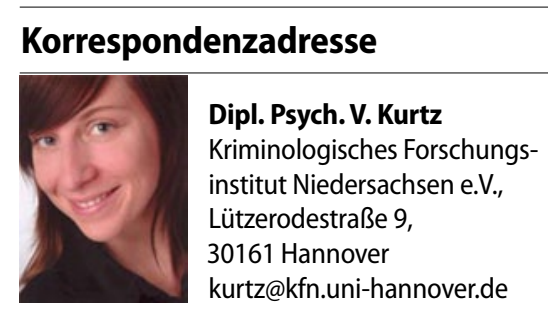

